\title{
Saccharin: the risks and benefits
}

Bernard L. Cohen of the University of Pittsburgh argues that the decision on whether or not to ban saccharin should be taken in the light of risk-benefit analysis. The risk of cancer may be outweighed by the risks of obesity.

SCIENTIFIC decision-making in matters of public concern should be made on the basis of risk-benefit analysis; but in the controversy over the banning of saccharin there has been little evidence that this has been done. That controversy involves decision making by the general public, so it is important that the public understand the risks in some quantitative fashion.

Since the average person does not readily accept numbers, the best substitute is to compare the risks of saccharin with other risks with which members of the public are familiar. It is my purpose here to give both a risk-benefit analysis and to compare saccharin risks with other risks familiar in our daily lives. All calculations will assume a linear relationship between dose and effects as is customary in estimates of this type.

In the recent Canadian study of bladder-cancer patients (Lancet, 17 September 1977, page 578), a possible link was established between that disease and the use of saccharin such that if the United States population $\left(2 \times 10^{8}\right)$ were to ingest one diet soft drink each day throughout their lives, there would be an extra 1,200 bladder cancers per year. This implies a risk of 1,200 cancers per $7.3 \times 10^{10}$ drinks, or one cancer per $6 \times 10^{7}$ drinks. There is ordinarily a time delay of 10 to 50 years between ingestion of a carcinogen and development of a cancer, so an average case would result in no more than a 20 year loss of life expectancy; thus, based on the results of the Canadian study, an average diet drink would reduce life expectancy by $20 \mathrm{yr} / 6 \times 10^{7}$, or about $9 \mathrm{~s}$.

To give a perspective on this number, smoking a single cigarette reduces life expectancy by $12 \mathrm{~min}$ (B. L. Cohen American Scientist 64,$550 ; 1977$ ) so a diet soft drink is about 80 times less dangerous than a cigarette. From the above result (or from the original finding) it is straightforward to calculate that one diet drink per day throughout life causes a reduction in life expectancy, $\Delta L$, of $2 \mathrm{~d}$; or

$$
\Delta L=2 \mathrm{~d}\left(\frac{\text { diet drinks }}{\mathrm{d}}\right)
$$

The benefits of diet soft-drinks result from their use in weight control by reducing calorific intake. Being overweight is well known to reduce life expectancy. In a rather old study, Pauling (Proc. Natl. Acad. Sri. 44,619;1958) analysed the data on this in a 1952 report of the Metropolitan Life Insurance Co. to obtain best fits to both a linear and quadratic relationship between loss of life expectancy, $L$, and overweight $\left(W-W_{0}\right)$, where $W$ is the weight and $W_{0}$ is the optimal weight. These were,

$$
\begin{aligned}
& L=17 \mathrm{yr}\left[\left(W-W_{\mathrm{o}}\right) / W_{\mathrm{o}}\right] \\
& L=36 \mathrm{yr}\left[\left(W-W_{\mathrm{o}}\right) / W_{\mathrm{o}}\right]^{2}
\end{aligned}
$$

Differentiating (3) and assuming that an average saccharin user would be at least $10 \%$ overweight and would weigh perhaps $160 \mathrm{lb}$ gives

$$
\Delta L=0.05 \mathrm{yr} / \mathrm{lb} \cdot \Delta W
$$

Applying the same assumptions to (2) gives

$$
\Delta L=0.11 \mathrm{yr} / \mathrm{lb} \cdot \Delta W
$$

independent of the percentage overweight.

There is more recent data on this subject from the same source (Metropolitan Life Insurance Co. Statistical Bulletin 10; 1960). To quote typical figures, for a 45 -year-old male with 150 lb optimal weight, weighing $170 \mathrm{lb}$ reduces life expectancy by
1.5 years and weighing $200 \mathrm{lb}$ reduces it by 4 years. This gives an approximately linear relationship with

$$
\begin{aligned}
\Delta L & =0.08 \mathrm{yr} / \mathrm{lb} . \Delta W \\
& =29 \mathrm{~d} / \mathrm{lb} \cdot \Delta W
\end{aligned}
$$

Since this is intermediate between (4) and (5) and is based on better data, I shall use (6).

An average person's body weight is related to his average daily calorie intake at about $1 \mathrm{lb}$ per 14 calories per day. Multiplying this by (6) gives a change in life expectancy

$$
\Delta L=2 \mathrm{~d} / \text { calories-per-d-intake }
$$

From a comparison between (1) and (7) we see that diet soft drinks give a net benefit if one such drink reduces calorific intake by more than I calorie.

There seems to be no firm evidence on the amount by which diet drinks reduce calorific intake (or body weight). A non-diet drink has about 100 calories, so if all other things were unchanged, substituting diet for non-diet drinks increases life expectancy by 100 times more than the cancer risk reduces it. This is perhaps an extreme assumption, but it would be very difficult to prove that it over-estimates the reduction in calorie intake from diet drinks by a factor of 100 . Unless this is done, there is no proof that the risk from diet drinks is greater than their benefits, and in fact it seems most likely that the opposite is the case.

If, on the other hand, we ignore the foregoing discussion and assume that there are no benefits from use of saccharin, it becomes important to understand the risks. We have already noted that ingesting a diet drink is 80 times less dangerous than smoking a cigarette, and we discuss here some other comparisons.

Perhaps the simplest risks to quantify are those due to automotive traffic. Approximately 8,000 people per year are killed in crossing streets in the United States. If the average American crosses five streets per day $\left(3.5 \times 10^{11}\right.$ crossings per year $)$, the risk per crossing is $8,000 / 3.5 \times 10^{11}$ or $2.3 \times 10^{-8}$. The risk from a diet drink is $1 / 6 \times 10^{-7}$, or $1.6 \times 10^{-8}$. Thus an average street crossing is about as dangerous as ingesting a diet drink.

Riding in automobiles involves a death risk of $2 \times 10^{-3}$ per mile; thus a diet drink is as dangerous as one mile of automobile travel. Using a small car rather than a large car approximately doubles the risk. So purchasing a small car rather than a standard size car is at least ten times as dangerous (assuming 30 miles per day average driving) as ingesting one diet drink per day during the period of ownership.

Perhaps the best understood cancer risk is that of women not having an annual test for cervical cancer. The mortality risk from this neglect is about $6 \times 10^{-7}$ per day, about 30 times higher than the risk of ingesting one diet drink per day.

The risk of one diet drink per day throughout life is readily calculated, using the Canadian study results, as $\left(1200 / 2 \times 10^{8}\right)$ risk per $\mathrm{yr} \times 70 \mathrm{yrs}=4 \times 10^{-1}$. Statistics show that the cancer risk varies considerably from one area of the country to another. For example, the risk is about 0.19 in New England compared to 0.15 in the south-east which has a similar age distribution. Thus moving from the south-east to New England involves 100 times greater cancer risk than ingesting one diet drink per day throughout life.

Approximately 7,000 Americans die each year due to fires, and it is estimated that about half of these could be saved if people would install smoke alarms in their homes (cost-about $\$ 5$ per year). Failure to install a smoke alarm is thus as dangerous as ingesting three diet drinks per day.

An endless list of comparisons could be put forward, but we have offered here a few examples that may be useful in helping understand the risk of saccharin. 